BUTP-98/25

$\mathrm{MPI} / \mathrm{PhT}-98-69$

hep-ph/9809468

\title{
Radiative Corrections in Inclusive Rare B Decays *
}

\author{
Christoph Greub and Tobias Hurth ${ }^{\mathrm{b}}$ \\ anstitute of Theoretical Physics, University of Bern \\ Sidlerstrasse 5, CH-3012 Bern, Switzerland \\ ${ }^{\mathrm{b}}$ Max-Planck-Institute for Physics, Werner-Heisenberg-Institute \\ Föhringer Ring 6, D-80805 Munich, Germany
}

We report recent theoretical progress in the analysis of radiative corrections in inclusive rare B-decays. In view of the B-factories, currently under construction at SLAC and KEK, and of the upgraded CESR experiment, the experimental status of rare B decays is expected to improve significantly in the near future. We review the complete NLL QCD calculations of the inclusive $b \rightarrow s \gamma$ and the $b \rightarrow d \gamma$ decay rates. We also discuss recently computed electroweak corrections and further improvements which lead to the current theoretical prediction of the $b \rightarrow s \gamma$ decay rate of $\mathcal{B}\left(B \rightarrow X_{s} \gamma\right)=(3.32 \pm 0.30) \times 10^{-4}$. We shortly comment on the theoretical uncertainty and on implications to physics beyond the SM. We collect the experimental data already available from CESR and LEP and discuss experimental and theoretical problems regarding the photon energy spectrum.

\section{Introduction}

In the Standard model (SM) rare B meson decays are induced by one-loop $\mathrm{W}$ exchange diagrams; therefore, new contributions where some of the SM particles in the loop are replaced by nonstandard particles like a charged Higgs boson, a gluino or a chargino, are not suppressed by an extra factor $\alpha / 4 \pi$ relative to the standard model amplitude. The resulting sensitivity for nonstandard contributions implies the possibility for an indirect observation of new physics, a strategy complementary to the direct production of new particles. The $B \rightarrow X_{s} \gamma$ decay, for example, plays an important role in restricting the parameter space of extensions of the SM like the minimal

\footnotetext{
*based on an invited talk given by T.H. at the International Euroconference on Quantum Chromodynamics (QCD 98), Montpellier, France, 2-8 July 1998.
}

supersymmetric standard model (MSSM) [1,2], in spite of the fact that the accuracy of the experimental data on $\mathcal{B}\left(B \rightarrow X_{s} \gamma\right)$ used for such analyses is not better than $30 \%$ at present. In this respect, also the $B \rightarrow X_{d} \gamma$ decay is of specific interest because its Cabibbo-Kobayashi-Maskawa (CKM) suppression by the factor $\left|V_{t d}\right|^{2} /\left|V_{t s}\right|^{2}$ in the SM may not be true in extended models.

Within the framework of the SM, rare B decays are important for constraining the CKM matrix elements: For example the $b \rightarrow s \gamma$ mode can be used to extract $\left|V_{t s}\right|$. The is possibly the most direct measurement of this CKM matrix element, as the decay mode $t \rightarrow W^{+} s$ is difficult to measure. Analogously, a future measurement of the $B \rightarrow X_{d} \gamma$ decay rate will help to drastically reduce the presently allowed region of the CKMWolfenstein parameters $\rho$ and $\eta$.

In contrast to exclusive decay channels, inclu- 
sive decay modes are theoretically clean in the sense that no specific model is needed to describe the final hadronic state. Nonperturbative effects in the inclusive modes are well under control due to heavy quark effective theory. For example, the decay width $\Gamma\left(B \rightarrow X_{s} \gamma\right)$ is well approximated by the partonic decay rate $\Gamma\left(b \rightarrow X_{s} \gamma\right)$ which can be analyzed in renormalization group improved perturbation theory. The class of non-perturbative effects which scales like $1 / m_{b}^{2}$ is expected to be well below $10 \%$ ([3], see also [4]). This numerical statement is supposed to hold also for the nonperturbative contributions which scale like $1 / m_{c}^{2}$ [5.

The accuracy in the dominating perturbative contribution was recently improved to next-toleading precision 66 13]: The renormalization scale dependence of the previous leading-log result at the $\pm 25 \%$-level was substantially reduced to $\pm 6 \%$ and the central value increased by about $20 \%$.

Much of the theoretical improvements carried out in the context of the decay $B \rightarrow X_{s} \gamma$ can straightforwardly adapted for the decay $B \rightarrow$ $X_{d} \gamma$. Like for the former decay, the NLLimproved and power-corrected decay rate for $B \rightarrow X_{d} \gamma$ has much reduced theoretical uncertainty which would allow to extract more precisely the CKM parameters from the measured branching ratio.

Finally, we mention that studies of direct $\mathrm{CP}$ violation in inclusive $B \rightarrow X_{s} \gamma$ and $B \rightarrow X_{d} \gamma$ decays in the SM and in its extensions have been presented recently 14.15.

The rest of the article is organized as follows. In section 2, we collect the experimental data recently presented by CLEO and ALEPH and discuss the problems regarding the photon energy spectrum. In section 3 , we recall the theoretical framework in which rare $\mathrm{B}$ decays can be analyzed and discuss the principle steps of the complete NLL QCD calculation of the inclusive $b \rightarrow s \gamma$ and $b \rightarrow d \gamma$ modes. In section 4 , we review electroweak corrections and further refinements which lead to the current theoretical prediction of the $B \rightarrow X_{s} \gamma$ decay rate. We shortly discuss the theoretical uncertainty and comment on bounds on physics beyond the SM.

\section{Experimental Data from CLEO and ALEPH, Photon Spectrum}

With the B-factories, presently under construction at SLAC (Babar) and KEK (Belle), and also with the upgraded B-factory at CESR in Cornell (CLEO III), the experimental situation regarding rare $\mathrm{B}$ decays will drastically change in the near future. With the expected high luminosity, radiative $\mathrm{B}$ decays will no longer be rare events. Experimental accuracy of below $10 \%$ in the inclusive $b \rightarrow s \gamma$ mode appears to be possible and even the measurement of exclusive $b \rightarrow d \gamma$ modes could be in reach. This was the motivation to increase also the accuracy of the theoretical prediction correspondingly.

However, experimental data is already available (for a review see [16,17]): In 1993, the first evidence for a penguin induced $\mathrm{B}$ meson decay was found by the CLEO collaboration. At the CESR $e^{+} e^{-}$storage ring, which operates just above the $B \bar{B}$ threshold at the $\Upsilon(4 S)$ resonance, they measured the exclusive electromagnetic penguin process $B \rightarrow K^{*} \gamma$. The inclusive analogue $B \rightarrow X_{s} \gamma$ was also found by the CLEO collaboration through the measurement of its characteristic photon energy spectrum in 1994. As this process is dominated by the two-body decay $b \rightarrow s \gamma$, its photon energy spectrum is expected to be a smeared delta function centered at $E_{\gamma} \approx m_{b} / 2$, where the smearing is due to perturbative gluon Bremsstrahlung and due to the non-perturbative Fermi motion of the b quark within the B meson. Some lower cutoff in the photon energy has to be imposed in order to exclude the background, mainly from the non-leptonic charged current processes $b \rightarrow c q \bar{q}^{\prime}+\gamma$ or $b \rightarrow u q \bar{q}^{\prime}+\gamma$, which have a typical Bremsstrahlung spectrum being maximal at $E_{\gamma}=0$ and falling off for larger values of $E_{\gamma}$. Therefore only the "kinematic" branching ratio for $B \rightarrow X_{s} \gamma$ in the range between $E_{\gamma}=2.2 \mathrm{GeV}$ and the kinematic endpoint at $E_{\gamma}=2.7 \mathrm{GeV}$ could be measured directly. To obtain from this measurement the total branching ratio, one has to know the fraction $R$ of the $B \rightarrow X_{s} \gamma$ events with $E_{\gamma} \geq 2.2 \mathrm{GeV}$. This was done in [6] where the Fermi motion of the b quark in the $\mathrm{B}$ meson was taken into account by us- 
ing the phenomenological model by Altarelli et al. (ACCMM model) 19]. Using this theoretical input regarding the photon energy spectrum, the value $R=0.87 \pm 0.06$ was used for the fraction by the CLEO collaboration, leading to the CLEO branching ratio 18

$\mathcal{B}\left(B \rightarrow X_{s} \gamma\right)=(2.32 \pm 0.57 \pm 0.35) \times 10^{-4}$

Actually, there are two separate CLEO analyses. The first technique constructs the inclusive rate by summing up the possible exclusive final states. Background in the measurement of exclusive modes is naturally low because of kinematical constraints and of the beam energy constraint. In the second technique one measures the inclusive photon spectrum near the end point. Background suppression is more difficult. For this purpose one uses topological differences between the spherical $B \bar{B}$ events and the two jets $e^{+} e^{-} \rightarrow q \bar{q}$. But the signal efficiency $(32 \%)$ is very high compared to the first technique. The branching ratio stated above (1) is the average of the two measurements, taking into account the correlation between the two techniques. The first error is statistical and the second is systematic (including model dependence). The measurement is based on a sample of $2.2 \times 10^{6} B \bar{B}$ events.

This summer (1998), CLEO has presented an improved, but preliminary measurement [20] which is based on $53 \%$ more data $\left(3.3 \times 10^{6}\right.$ events). They also used the slightly wider $E_{\gamma}$ window starting at $2.1 \mathrm{GeV}$. The relative error drops almost by a factor of $\sqrt{3}$ :

$$
\mathcal{B}\left(B \rightarrow X_{s} \gamma\right)=(3.15 \pm 0.35 \pm 0.32 \pm 0.26) \times 10^{-4}(2)
$$

The errors represent statistics, systematics, and the model dependence, respectively. In ref. [20] the kinematical branching fraction $\mathcal{B}^{2.1}$ for photons with energies in the range between $2.1 \mathrm{GeV}$ and $2.7 \mathrm{GeV}$ is also given:

$\mathcal{B}^{2.1}\left(B \rightarrow X_{s} \gamma\right)=(2.97 \pm 0.33 \pm 0.30 \pm 0.21) \times 10^{-4}(3)$

As CLEO II still analyses more data, one can expect an even better measurement soon.

There is also data at the $Z^{0}$ peak from the LEP experiments. The ALEPH collaboration [21] has measured the inclusive branching ratio

$\mathcal{B}\left(H_{b} \rightarrow X_{s} \gamma\right)=(3.11 \pm 0.80 \pm 0.72) \times 10^{-4}$.
It should be noted that the branching ratio in (4) involves a different weighted average of the B mesons and $\Lambda_{b}$ baryons produced in $Z^{0}$ decays (hence the symbol $H_{b}$ ) than the corresponding one given by CLEO. High luminosity is more difficult to obtain at higher $e^{+} e^{-}$collision energies. Thus, $B \bar{B}$ samples obtained by the LEP experiments are smaller than the one at CESR. The rate measured by ALEPH, is consistent with the CLEO measurement.

The uncertainty regarding the fraction $R$ of the $B \rightarrow X_{s} \gamma$ events with $E_{\gamma} \geq 2.2 \mathrm{GeV}$ spotted in the experimental measurement should be regarded as a purely theoretical uncertainty. As mentioned above, the fraction $R$ was calculated in [6] using the phenomenological model by Altarelli et al., where the Fermi motion of the b quark in the $\mathrm{B}$ meson is characterized by two parameters, the average Fermi momentum $p_{F}$ of the $\mathrm{b}$ quark and the mass $m_{q}$ of the spectator quark. The error on the fraction $R$ is essentially obtained by varying the model parameters $p_{F}$ and $m_{q}$ in the range for which the ACCMM model correctly describes the energy spectrum of the charged lepton in the semileptonic decays $B \rightarrow X_{c} \ell \nu$ and $B \rightarrow X_{u} \ell \nu$, measured by CLEO and ARGUS. In [6] a first comparison between the calculated photon energy spectrum and the one measured by the CLEO collaboration was presented. The (normalized) measured photon energy spectrum and the theoretical one are in agreement for those values of $p_{F}$ and $m_{q}$, which correctly describe the inclusive semileptonic CLEO data $B \rightarrow X_{c} \ell \nu$ and $B \rightarrow X_{u} \ell \nu$; at present, the data from the radiative decays is, however, not precise enough to further constrain the values of $p_{F}$ and $m_{q}$. The best fit between the theoretical and measured photon energy spectrum is obtained for $p_{F}=450 \mathrm{MeV}$ and $m_{q}=0$.

Besides this phenomenological model by Altarelli et al., more fundamental theoretical methods are available today to implement the bound state effects, namely by making use of operator product expansion techniques in the framework of heavy quark effective theory (HQET). A new analysis along these lines was recently presented [22]. Unfortunately, the operator product expansion breaks down near the endpoint of the 
photon energy spectrum and therefore an infinite number of leading-twist corrections has to be resummed into a non-perturbative universal "shape function" which determines the light-cone momentum distribution of the b-quark in the B meson. The physical decay distributions are then obtained from a convolution of parton model spectra with this shape function. At present this function cannot be calculated, but there is at least some information on the moments of the shape function which are related to the forward matrix elements of local operators. Ansätze for the shape function, constrained by the latter information, are used. In contrast to the older analysis based on the ACCMM model, the new analysis of Kagan and Neubert 22] includes the full NLL information. Their fraction $R=0.78_{-0.11}^{+0.09}$ (for the energy cut $E_{\gamma}>2.2 \mathrm{GeV}$ ) is significantly smaller than the factor used by CLEO. The larger error on $R$ implies that the theoretical uncertainty in the calculation of Fermi motion effects has been underestimated until now. Clearly, a lower experimental cut decreases the sensitivity to the parameters of the shape function (or, more general, the model dependence as one can already see from the new CLEO measurement.) Another future aim should be to determine the shape function (and analogously the parameter of the ACCMM model) by using the high-precision measurements of the photon energy spectrum.

\section{QCD Corrections at the NLL level}

Short distance QCD corrections enhance the partonic decay rate $\Gamma(b \rightarrow s \gamma)$ by more than a factor of two. These QCD effects bring in large logarithms of the form $\alpha_{s}^{n}\left(m_{b}\right) \log ^{m}\left(m_{b} / M\right)$, where $M=m_{t}$ or $M=m_{W}$ and $m \leq n$ (with $n=$ $0,1,2, \ldots)$. This is a natural feature in any process where two different mass scales are present. In order to get a reasonable result, one has to resum at least the leading-log (LL) series $(m=n)$. Working to next-to-leading-log (NLL) precision means that one is also resumming all the terms of the form $\alpha_{s}\left(m_{b}\right)\left(\alpha_{s}^{n}\left(m_{b}\right) \ln ^{n}\left(m_{b} / M\right)\right)$.

The error of the leading logarithmic (LL) result 23. was dominated by a large renormalization scale dependence at the $\pm 25 \%$ level which in- dicates the importance of the NLL series. Moreover, such a NLL program is also important in order to ensure validity of renormalization group improved perturbation theory in the example under question. It was recently found in Multi-Higgs Doublet Models [24] that the truncation of the perturbative series at the NLL level is often not appropriate.

A suitable framework to achieve the necessary resummations of the large logs is an effective lowenergy theory, obtained by integrating out the heavy particles which in the SM are the top quark and the $W$-boson. The effective Hamiltonian relevant for $b \rightarrow s \gamma$ and $b \rightarrow s g$ in the SM and many of its extensions reads

$H_{e f f}(b \rightarrow s \gamma)=-\frac{4 G_{F}}{\sqrt{2}} \lambda_{t} \sum_{i=1}^{8} C_{i}(\mu) O_{i}(\mu)$

where $O_{i}(\mu)$ are the relevant operators, $C_{i}(\mu)$ are the corresponding Wilson coefficients, which contain the complete top- and W- mass dependence, and $\lambda_{t}=V_{t b} V_{t s}^{*}$ with $V_{i j}$ being the CKM matrix elements. The CKM dependence globally factorizes, because we work in the approximation $\lambda_{u}=0\left(\right.$ for $\left.B \rightarrow X_{s} \gamma\right)$.

Neglecting operators with dimension $>6$ which are suppressed by higher powers of $1 / m_{W / t}$ and using the equations of motion for the operators, one arrives at the following basis of dimension 6 operators

$$
\begin{aligned}
O_{1} & =\left(\bar{c}_{L \beta} \gamma^{\mu} b_{L \alpha}\right)\left(\bar{s}_{L \alpha} \gamma_{\mu} c_{L \beta}\right), \\
O_{2} & =\left(\bar{c}_{L \alpha} \gamma^{\mu} b_{L \alpha}\right)\left(\bar{s}_{L \beta} \gamma_{\mu} c_{L \beta}\right), \\
O_{7} & =\frac{e}{16 \pi^{2}} \bar{s}_{\alpha} \sigma^{\mu \nu}\left(m_{b}(\mu) R\right) b_{\alpha} F_{\mu \nu}, \\
O_{8} & =\frac{g_{s}}{16 \pi^{2}} \bar{s}_{\alpha} \sigma^{\mu \nu}\left(m_{b}(\mu) R\right) \frac{\lambda_{\alpha \beta}^{A}}{2} b_{\beta} G_{\mu \nu}^{A}
\end{aligned}
$$

Because the Wilson coefficients of the penguin induced Four-Fermi operators $O_{3}, . . O_{6}$ are very small, we do not list them here.

In this framework the next-to-leading logarithmic terms $\alpha_{s}\left(m_{b}\right)\left(\alpha_{s}^{n}\left(m_{b}\right) \log ^{n}\left(m_{b} / m_{W / t}\right)\right)$ in the $b \rightarrow s \gamma$ amplitude have two sources:

1 - The corrections to the Wilson coefficients $C_{i}(\mu)$ at the scale $\mu \approx m_{b}$.

2 - The corrections to the matrix elements of the operators $O_{i}$ also at the low-energy scale $\mu \approx m_{b}$. 
Only the sum of the two contributions is renormalization scheme independent and in fact, from the $\mu$-independence of the effective Hamiltonian, one can derive a renormalization group equation (RGE) for the Wilson coefficients $C_{i}(\mu)$ :

$\mu \frac{d}{d \mu} C_{i}(\mu)=\gamma_{j i} C_{j}(\mu) \quad$,

where the $(8 \times 8)$ matrix $\gamma$ is the anomalous dimension matrix of the operators $O_{i}$. The standard procedure to calculate the two contributions involves the following three steps:

ad 1a $\bullet$ One matches the full standard model theory with the effective theory at the scale $\mu=\mu_{W}$, where $\mu_{W}$ denotes a scale of order $m_{W}$ or $m_{t}$. At this scale, the matrix elements of the operators in the effective theory lead to the same logarithms as the full theory calculation. Consequently, the Wilson coefficients $C_{i}\left(\mu_{W}\right)$ only pick up small QCD corrections, which can be calculated in fixed-order perturbation theory. In the LL (NLL) program, the matching has to be worked out to order $\alpha_{s}^{0}\left(\alpha_{s}^{1}\right)$.

ad 1b - Solving the RGE (77) and using the $C_{i}\left(\mu_{W}\right)$ of Step 1a as initial conditions, one performs the evolution of these Wilson coefficients from $\mu=\mu_{W}$ down to $\mu=\mu_{b}$, where $\mu_{b}$ is of the order of $m_{b}$. As the matrix elements of the operators evaluated at the low scale $\mu_{b}$ are free of large logarithms, the latter are contained in resummed form in the Wilson coefficients. For a LL (NLL) calculation, this RGE step has to be performed using the anomalous dimension matrix $\gamma_{j i}$ up to order $\alpha_{s}^{1}\left(\alpha_{s}^{2}\right)$.

ad 2 - The corrections to the matrix elements of the operators $\left\langle s \gamma\left|O_{i}(\mu)\right| b\right\rangle$ at the scale $\mu=\mu_{b}$ have to be calculated to order $\alpha_{s}^{0}\left(\alpha_{s}^{1}\right)$ in the LL (NLL) calculation.

All three steps $(1 \mathrm{a}, 1 \mathrm{~b}, 2)$ to NLL precision involve rather difficult calculations. The most difficult part in Step 1a is the two-loop (or order $\alpha_{s}$ ) matching of the dipole operators $O_{7}$ and $O_{8}$. It involves two-loop diagrams both in the full and in the effective theory. It was worked out by Adel and Yao [7] some time ago. As this is a crucial step in the NLL program, Greub and Hurth confirmed their findings in a detailed recalculation using a different method [11]. Re- cently, two further recalculations of this result were presented [12,13]. Step 2 basically consists of Bremsstrahlung corrections and virtual corrections. While the Bremsstrahlung corrections were worked out some time ago by Ali and Greub [6] and have been confirmed and extended by Pott [8]. A complete analysis of the virtual two-loop corrections (up to the contributions of the FourFermi operators with very small coefficients) was presented by Greub, Hurth and Wyler [9]. The order $\alpha_{s}^{2}$ anomalous matrix (Step 1b) has been worked out by Chetyrkin, Misiak and Münz [10]. The extraction of some of the elements in the $O\left(\alpha_{s}^{2}\right)$ anomalous dimension matrix involves pole parts of three-loop diagrams.

Combining the NLL calculations of all the three steps $(1 \mathrm{a}+\mathrm{b}, 2)$, the first theoretical prediction to NLL precision for the branching ratio was presented in [10]:

$B R\left(B \rightarrow X_{s} \gamma\right)=(3.28 \pm 0.33) \times 10^{-4}$.

The theoretical error has two dominant sources: The $\mu$ dependence is reduced to about $6 \%$. The other main uncertainty of $5 \%$ stems from the $m_{c} / m_{b}$ dependence. This first theoretical NLL prediction already included the nonperturbative correction which scale with $1 / m_{b}^{2}$ which are rather small at the $1 \%$ level. Later, also nonperturbative contributions from $c \bar{c}$ intermediate states were considered which scale with $1 / m_{c}^{2}$ [5]. Detailed investigations [5] show that these contributions enlarge the branching ratio by $3 \%$.

The $B \rightarrow X_{d} \gamma$ decay can be treated analogously. The effective Hamiltonian is the same in the processes $b \rightarrow s \gamma(+g)$ and $b \rightarrow d \gamma(+g)$ up to the obvious replacement of the $s$-quark field by the $d$-quark field. However, as $\lambda_{u}$ for $b \rightarrow d \gamma$ is not small relative to $\lambda_{t}$ and $\lambda_{c}$, one also has to encounter the operators proportional to $\lambda_{u}$. The matching conditions $C_{i}\left(m_{W}\right)$ and the solutions of the RG equations, yielding $C_{i}\left(\mu_{b}\right)$, coincide with those needed for the process $b \rightarrow s \gamma(+g)$. The power corrections in $1 / m_{b}^{2}$ and $1 / m_{c}^{2}$ (besides the CKM factors) are also the same for both modes. However, the so-called long-distance contributions from the intermediate $u$-quark in the penguin loops are different. These are suppressed in the $B \rightarrow X_{s} \gamma$ mode due to the un- 
favorable CKM matrix elements. In $B \rightarrow X_{d} \gamma$, however, there is no CKM-suppression and one has to include the long-distance intermediate $u$ quark contributions. The non-perturbative contribution generated by the $u$-quark loop can only be modeled at present.

\section{Theoretical Predictions, Current Status}

The prediction for the partonic $b \rightarrow s \gamma$ decay rate is usually normalized by the semileptonic decay rate in order to get rid of uncertainties related to the fifth power of the b quark mass. Moreover, often an explicit lower cut on the photon energy in the Bremsstrahlung correction is made:

$R_{\text {quark }}(\delta)=\frac{\Gamma[b \rightarrow s \gamma]+\Gamma[b \rightarrow s \gamma \text { gluon }]_{\delta}}{\Gamma\left[b \rightarrow X_{c} e \bar{\nu}_{e}\right]}$

where the subscript $\delta$ means that only photons with energy $E_{\gamma}>(1-\delta) E_{\gamma}^{\max }=(1-\delta) \frac{m_{b}}{2}$ are counted. The ratio $R_{\text {quark }}$ is divergent in the limit $\delta \rightarrow 1$ due to the unphysical soft photon divergence in the subprocess $b \rightarrow$ sqgluon. In this limit only the sum of $\Gamma[b \rightarrow s \gamma], \Gamma[b \rightarrow$ sgluon $]$ and $\Gamma[b \rightarrow$ sqgluon $]$ is a reasonable physical quantity, in which all divergences cancel out. In [9] the limit $\delta \rightarrow 1$ is taken and the singularities are removed by adding the virtual photon corrections to $b \rightarrow$ sgluon. In 10 the "total" partonic $b \rightarrow s \gamma$ is defined by using the value at $\delta=0.99$. However, in [22] it was shown that this is not the most suitable choice, because the theoretical result becomes more sensitive to the unphysical soft-photon divergence than the first numerical results indicated. The authors of 22] use $\delta=0.90$ as their optimized definition of the total decay rate. They also suggest to directly compare theory and experiment using the same energy cuts as $\operatorname{CLEO}\left(E_{\gamma}>2.1(2.2) \mathrm{GeV}\right)$. Then the theoretical uncertainty regarding the photon energy spectrum discussed in section 2 would occur naturally in the theoretical prediction.

Using the measured semileptonic branching ratio $\mathcal{B}_{\text {exp. }}^{\text {sl }}$, the branching ratio $\mathcal{B}\left(B \rightarrow X_{s} \gamma\right)$ is given by

$\mathcal{B}\left(B \rightarrow X_{s} \gamma\right)=R_{\text {quark }} \times \mathcal{B}_{\text {exp. }}^{\text {sl }}\left(1+\Delta_{\text {nonpert }}\right)(10)$ where $\Delta_{\text {nonpert }}$ contains the $1 / \mathrm{m}_{b}^{2}$ and $1 / \mathrm{m}_{c}^{2}$ corrections, whose effects amount to $+1 \%$ and $+3 \%$, respectively.

The NLL QCD analysis for the branching ratio $\mathcal{B}\left(B \rightarrow X_{s} \gamma\right)$ is further based on the following values for the input parameters [24]: $\alpha_{s}\left(M_{Z}\right)=$ $0.119 \pm 0.004, m_{b}-m_{c}=3.39 \pm 0.04 \mathrm{GeV}$, $m_{c} / m_{b}=0.29 \pm 0.02, m_{t}($ pole $)=(175 \pm 5) \mathrm{GeV}$, $\mathcal{B}_{s l}=(10.49 \pm 0.46) \%, \alpha_{e m}^{-1}=(130.3 \pm 2.3)$. For the CKM matrix factor $\left|V_{t s}^{*} V_{t b} / V_{c b}\right|^{2}$ the number $(0.95 \pm 0.03)$ is used. For these values of the input parameters, one gets

$\mathcal{B}\left(B \rightarrow X_{s} \gamma\right)=(3.57 \pm 0.32) \times 10^{-4}$.

The shift in the central value compared with the prediction (8) has different sources. The recently discovered nonperturbative corrections scaling with $m_{c}^{2}$ are included in addition. Besides slightly different input parameters, the discussed convention $\delta \rightarrow 1$ is used (11), while in (8) $\delta=0.99$ was chosen. The central value is also sensitive to the details how the uncalculated next-next-leading order terms are discarded. We note that in (11) the factor $1 / \Gamma_{s l}$ is not expanded in $\alpha_{s}$. (If one does so, the central value in (11) decreases from $3.57 \times 10^{-4}$ to $3.46 \times 10^{-4}$.)

We emphasize that for a comparison with the ALEPH measurement (4) one should use consistently the measured semileptonic branching ratio $\mathcal{B}\left(H_{b} \rightarrow X_{c, u} \ell \nu\right)=(11.16 \pm 0.20) \%$. This leads to a larger theoretical prediction for the LEP experiments.

Quite recently, A. Czarnecki and W. Marciano [25] calculated part of the electroweak two-loop contributions, namely contributions from fermion loops in gauge boson propagators $(\gamma$ and $W$ ) and from short-distance photonic loop corrections which are considered to be the two dominant classes of electroweak corrections. They found that these new contributions reduce the $R_{\text {quark }}$ (11) by $9 \%$. They observed that the on-shell value of the fine structure constant $1 / \alpha_{e m}=137$ is more appropriate for real photon emission instead of the value $1 / \alpha_{e m}=(130.3 \pm 2.3)$ used in previous analyses. Their loop calculations confirmed this expectation. This change in $\alpha_{e m}$ represents the main reduction of $-5 \%$ in $R_{\text {quark }}$. Also recently, Strumia 26] made a complete anal- 
ysis of the heavy top and the heavy Higgs corrections to $b \rightarrow s \gamma$ in the limit $m_{W} \rightarrow 0$. The correction is below $1 \%$ which indicates that the $-2.2 \%$ correction from the fermion loop contribution stated in [25] could be an overestimation. Kagan and Neubert 22] improved the QED analysis made in [25] by resumming the contributions of order $\alpha \ln \left(m_{W} / \mu_{b}\right)\left(\alpha_{s} \ln \left(m_{W} / \mu_{b}\right)^{n}\right.$ to all orders (while in 25] only the $n=0$ contribution was included). This resummation decreases the QED corrections.

Including only the resummed QED corrections, using the on-shell value of $\alpha_{e m}$ and working with the convention $\delta \rightarrow 1$ in $R_{\text {quark }}$, we end up with the current theoretical prediction

$\mathcal{B}\left(B \rightarrow X_{s} \gamma\right)=(3.32 \pm 0.14 \pm 0.26) \times 10^{-4}$,

where the first error represents the uncertainty regarding the scale dependences on $\mu_{b}$ and $\mu_{W}$, while the second error is the uncertainty due to the input parameters.

A complete NLL calculation of the $B \rightarrow$ $X_{s} \gamma$ branching ratio in the simplest extension of the SM, namely the Two-Higgs-Doublet Model $(2 \mathrm{HDM})$, was recently presented in 12,24]. In the 2HDM of Type II (which already represents a good approximation for gauge-mediated supersymmetric models with large $\tan \beta$ where the charged Higgs contribution dominates the chargino contribution) a lower bound on the mass of the charged Higgs boson of about $250 \mathrm{GeV}$ was found for large $\tan \beta$. This bound was based on the CLEO upper limit (95\% C.L.) of $4.2 \times 10^{-4}$ for $\mathcal{B}\left(B \rightarrow X_{s} \gamma\right)[18$ and the electromagnetic corrections discussed above were not included. Taking into account these corrections, and using the most recent upper CLEO bound $\mathcal{B}\left(B \rightarrow X_{s} \gamma\right)<$ $4.5 \times 10^{-4}$ (at $95 \%$ C.L.) [20], the lower bound for the mass of the charged Higgs bosons is at about $165 \mathrm{GeV}$ [27] for large $\tan \beta$. This value should be compared with the lower bound of 45 $\mathrm{GeV}$ found in the direct search for the charged Higgs boson at LEP-I. The validity of the bound at $\sim 55 \mathrm{GeV}$ from LEP-II was criticized in [28]. Quite recently, a more general SUSY scenario was presented [29], where in particular the possibility of destructive interference of the chargino and the charged Higgs contribution is analyzed.
Instead of making a theoretical standard model prediction for the branching ratio $\mathcal{B}\left(B \rightarrow X_{s} \gamma\right)$, one can use the experimental data and theory in order to directly determine the combination $\left|V_{t b} V_{t s}^{*}\right| /\left|V_{c b}\right|$ of CKM matrix elements; in turn, one can determine $\left|V_{t s}\right|$, by making use of the relatively well known CKM matrix elements $V_{c b}$ and $V_{t b}$. An update of the analysis done by A. Ali [30, using the CLEO data (2), the ALEPH data (1), and including all new contributions on the theoretical side, leads to

$\frac{\left|V_{t s}^{*} V_{t b}\right|}{\left|V_{c b}\right|}=0.95 \pm 0.08_{\text {exp. }} \pm 0.05_{t h .} \quad$ CLEO
$\frac{\left|V_{t s}^{*} V_{t b}\right|}{\left|V_{c b}\right|}=0.91 \pm 0.15_{\text {exp. }} \pm 0.04_{t h .} \quad$ ALEPH.

The average of the two measurements yields

$\frac{\left|V_{t s}^{*} V_{t b}\right|}{\left|V_{c b}\right|}=0.93 \pm 0.09 \pm 0.03=0.93 \pm 0.10$

where in the very last step the theoretical and experimental errors were added in quadrature. Using $\left|V_{t b}\right|=0.99 \pm 0.15$ from the CDF measurement and $\left|V_{c b}\right|=0.0393 \pm 0.0028$ extracted from semileptonic $B$ decays, one obtains

$\left|V_{t s}\right|=0.037 \pm 0.007$,

where all the errors were added in quadrature. This is probably the most direct determination of this CKM matrix element, as mentioned in the introduction. With an improved measurement of $\mathcal{B}\left(B \rightarrow X_{s} \gamma\right)$ and $V_{t b}$, one expects to reduce the present error on $\left|V_{t s}\right|$ by a factor of 2 or even more.

Let us finally move to the predictions for the $B \rightarrow X_{d} \gamma$ decay 15. One finds that for $\mu_{b}=2.5$ $\mathrm{GeV}$ (and the central values of the input parameters) the difference between the LL and NLL results is $\sim 10 \%$, increasing the branching ratio in the NLL case. For a fixed value of the CKM-Wolfenstein parameters $\rho$ and $\eta$, the theoretical uncertainty of the branching ratio is: $\Delta \mathcal{B}\left(B \rightarrow X_{d} \gamma\right) / \mathcal{B}\left(B \rightarrow X_{d} \gamma\right)= \pm(6-10) \%$. Of particular theoretical interest is the ratio of the branching ratios, defined as

$R(d \gamma / s \gamma) \equiv \frac{\mathcal{B}\left(B \rightarrow X_{d} \gamma\right)}{\mathcal{B}\left(B \rightarrow X_{s} \gamma\right)}$, 
in which a good part of the theoretical uncertainties cancels. As expected, the uncertainty in the ratio $R(d \gamma / s \gamma)$ is indeed smaller. This suggests that a future measurement of $R(d \gamma / s \gamma)$ will have a large impact on the CKM phenomenology.

Varying the CKM-Wolfenstein parameters $\rho$ and $\eta$ in the range $-0.1 \leq \rho \leq 0.4$ and $0.2 \leq$ $\eta \leq 0.46$ and taking into account other parametric dependences stated above, the results (without electroweak corrections) are

$$
\begin{aligned}
6.0 \times 10^{-6} & \leq \mathcal{B}\left(B \rightarrow X_{d} \gamma\right) \leq 2.6 \times 10^{-5} \\
0.017 & \leq R(d \gamma / s \gamma) \leq 0.074
\end{aligned}
$$

These quantities are expected to be measurable at the forthcoming high luminosity B facilities. At present only upper bounds on corresponding exclusive modes are available from CLEO II, namely $\mathcal{B}\left(B^{0} \rightarrow \rho^{0} \gamma\right)=<3.9 \cdot 10^{-5}, \mathcal{B}\left(B^{0} \rightarrow \omega \gamma\right)=<$ $1.3 \cdot 10^{-5}$, and $\mathcal{B}\left(B^{-} \rightarrow \rho^{-} \gamma\right)=<1.1 \cdot 10^{-5}$ 17.

\section{Summary}

Significant theoretical progress in the analysis of inclusive rare radiative B-decays has been achieved during the last few years. In particular, the completion of the NLL QCD calculation for the dominating perturbative contribution and further refinements on nonperturbative and electroweak corrections and on the photon energy spectrum lead to the present theoretical prediction of the $B \rightarrow X_{s} \gamma$ branching ratio with a substantially improved precision. These theoretical improvements call for more precise experimental data which one can expect from the B-factories and the upgraded CLEO experiment in the next years. This experimental data will provide important tests of the SM and its extensions.

\section{REFERENCES}

1. S. Bertolini, F. Borzumati, A. Masiero, and G. Ridolfi, Nucl. Phys. B 353 (1991) 591.

2. M. Gronau and D. London, Phys. Rev. D55 (1997) 2845; J.L. Hewett and J.D. Wells Phys. Rev. D55 (1997) 55.

3. A.F. Falk, M. Luke and M. Savage, Phys. Rev. D49 (1994) 3367.
4. A. Ali, G. Hiller, L.T. Handoko and T. Morozumi, Phys. Rev. D55 (1997) 4105.

5. M.B. Voloshin, Phys. Lett. B 397 (1997) 295; A. Khodjamirian, R. Rückl, G. Stoll and D. Wyler, Phys. Lett. B402 (1997) 167; Z. Ligeti, L. Randall, and M.B. Wise, Phys. Lett. B 402 (1997) 178; A.K. Grant, A.G. Morgan, S. Nussinov, and R.D. Peccei, Phys. Rev. D 56 (1997) 3151; G. Buchalla, G. Isidori, and S.J. Rey, Nucl. Phys. B 511 (1998) 594.

6. A. Ali and C. Greub, Z. Phys. C49 (1991) 431; Phys. Lett.B259 (1991) 182;Z. Phys. C60 (1993) 433; Phys. Lett. B361 (1995) 146.

7. K. Adel and. Y.-P. Yao, Phys. Rev. D49 (1994) 4945.

8. N. Pott, Phys. Rev. D54 (1996) 938.

9. C. Greub, T. Hurth and D. Wyler, Phys. Lett. B380 (1996) 385; Phys. Rev. D54 (1996) 3350 .

10. K. Chetyrkin, M. Misiak and M. Münz, Phys. Lett. B400 (1997) 206.

11. C. Greub and T. Hurth Phys. Rev. D 56 (1997) 2934.

12. M. Ciuchini, G. Degrassi, P. Gambino, and G.F. Giudice, hep-ph/9710335

13. A.J. Buras, A. Kwiatkowski, and N. Pott, hep-ph/9710336.

14. A. Kagan and M. Neubert, hep-ph/9803368.

15. A. Ali, A. Asatrian and C. Greub, hep$\mathrm{ph} / 9803314$.

16. M. Feindt, hep-ph/9802380.

17. K. Lingel, T. Skwarnicki and J.G. Smith, hep$\mathrm{ex} / 9804015$.

18. M.S. Alam et al. (CLEO Collab.), Phys. Rev. Lett. 74 (1995) 2885

19. G. Altarelli et al., Nucl. Phys. B 208 (1982) 365; A. Ali and E. Pietarinen, Nucl. Phys. B 154 (1979) 519.

20. M.S. Alam, CLEO CONF 98-17, contribution to the ICHEP98 conference, Vancouver 1998. J. Alexander, talk given at the ICHEP98 conference, Vancouver 1998.

21. R. Barate et al. (ALEPH Collab.) CERNEP/98-044 (1998).

22. A.Kagan and M.Neubert, hep-ph/9805303.

23. M. Ciuchini, E. Franco, G. Martinelli, L. Reina and L. Sivestrini, Phys. Lett. B316 
(1993) 127; Nucl. Phys. B415 (1994) 403. G. Cella, G. Curci, G. Ricciardi and A. Vicere, Phys. Lett. B325 (1994) 227. M. Misiak, Nucl. Phys. B393 (1993) 23; Erratum ibid. B439 (1995) 461.

24. F. Borzumati and C. Greub, hep-ph/9802391.

25. A. Czarnecki and W.J. Marciano, hep$\mathrm{ph} / 9804252$

26. A. Strumia, hep-ph/9804274.

27. F. Borzumati and C. Greub, hep-ph/9809438.

28. F. Borzumati, A. Djouadi, hep-ph/9806301.

29. M. Ciuchini, G. Degrassi, P. Gambino and G.F. Giudice, hep-ph/9806308.

30. A. Ali, DESY 97-192, hep-ph/9709507 v3. 